\title{
„Die Region ist tot - es lebe die Region!“
}

\author{
Anmerkungen zur Diskurskonjunktur und Relativierung des Begriffes* \\ "The Region is Dead - Long Live the Region!"
}

Remarks on the State of Scholarly Debate and on the Relativity of the Term "Region"

\begin{abstract}
Kurzfassung
Die in der räumlichen Planung seit einigen Jahren zu beobachtende inflationäre Verwendung des Regionsbegriffes geht einher mit einer neuen Unübersichtlichkeit räumlicher Bezugseinheiten. Da gerade unter dem Gesichtspunkt planerischer Gestaltung ïberzeugende Regionsbegriffe fehlen, ist die Raumwissenschaft aufgefordert, ein neues Regionenverständnis zu entwickeln. Der Autor vertritt hierbei die Auffassung, den Regionsbegriff nicht als physischen Ausschnitt der Erdoberfläche (miss)zu verstehen, sondern als sozio-ökonomischen Verflechtungsraum zu betrachten, der sich als lose verbundenes Netzwerk durch die gegenseitige Wahrnehmung der in ihm agierenden Akteure definiert.
\end{abstract}

\section{Abstract}

The inflationary use of the term "region", observable over the last few years in spatial planning circles, has been accompanied by a new sense of confusion in respect of spatial units. In the absence of convincing definitions of the term "region", at least of relevance to physical planning, the spatial sciences are called upon to develop a new conception of what is meant by "regions". The author takes the view that the region should not be (mis) understood simply as a physical section of the earth's surface, but should rather be viewed as an area characterised by socio-economic integration which defines itself as a loosely connected network through the mutual perception of the actors who operate within it.

„Die Begriffe in einer Wissenschaft sind die Träger des wissenschaftlichen Gebäudes und verhalten sich zu ihr selbst wie das Gerippe zu dem menschlichen Körper; sie geben ihm Festigkeit und Haltung."

Paul Johann Anselm Feuerbach (1804)

Die Region ist „in“. Zwar begann der Aufschwung des regionalen Denkens im westlichen Europa bereits in den 60er Jahren. ${ }^{1}$ Der endgültige Durchbruch zum Schlagwort von "fast mythischer Bedeutung “2 ist jedoch erst in den vergangenen Jahren zu beobachten. Nur wenige Begriffe konnten sich im öffentlichen Sprachgebrauch in den 90er Jahren eine vergleichbare Popularität sichern. Die Politik verwendet ihn heute ebenso selbstverständlich wie nahezu jede Wissenschaftsdisziplin. Er wird für vielfältige Zwecke proklamiert, in zahllose Konzepte integriert. Er ist allen modernen europäischen Sprachen gleichermaßen vertraut. Ein derart variabler Begriff muss allen einschlägi- gen Forschungsbemühungen zum Trotz diffus bleiben. Definitionsansätzen haftet zwangsläufig eine gewisse Beliebigkeit an.

In der traditionellen Geographie hatte der Regionsbegriff noch einen einigermaßen klaren Bedeutungsgehalt als real vorhandene geographisch-räumliche Einheit mittlerer Größenordnung. Nach diesem heute jedoch weitgehend obsoleten, positivistischen Verständnis waren Regionen „im Erdraum scheinbar unverrückbar fixierbare, in einem Atlas darstellbare Raumeinheiten“.3 Versuche, angesichts der Diskurskonjunktur des Regionalen einen neuen, methodisch und begrifflich reflektierten Umgang mit der Denkfigur „Region“ zu etablieren, setzen in den Raumwissenschaften erst allmählich ein. Dabei sind es neben politikwissenschaftlichen Sammelbänden v.a. geographische Abhandlungen, die die theoretische Diskussion in jüngster Zeit belebt haben und die als erste 
Schritte auf dem Weg zu einer umfassenderen „Theorie der Region" aufzufassen sind. ${ }^{4}$

So ist nach Weichhart für die inflationäre Verwendung des Regionsbegriffs der hinter diesem Terminus stehende charakteristische Abstraktions- und Generalisierungsprozess ausschlaggebend. ${ }^{5}$ Dieser Prozess der Hypostasierung oder Reifikation führe dazu, dass räumliche Interaktionszusammenhänge als gegenständliche Objekte behandelt würden. Mit anderen Worten: Vorstellungen über die Realität würden mit der Realität selbst verwechselt. Regionen sind für Weichhart je nach Interpretationszusammenhang sowohl Chimäre als auch Konstrukte oder auch Strukturprinzipien sozialer Systeme. Wie Weichhart kommt auch Blotevogel zu dem Schluss, dass Regionen stets einen konstruktivistischen Charakter aufweisen, also das Ergebnis einer spezifischen Regionalisierung sind. ${ }^{6}$ Der dadurch erwachsenen „neuen Unübersichtlichkeit" stünden neue Perspektiven, Fragen der „Regionsbildung“ und "Regionserfindung“ aber auch des Regionalmanagements gegenüber.

Die „Unübersichtlichkeit“ räumlicher Bezugseinheiten nimmt noch zu, bezieht man die Debatte um die „New Regional Geography“ der angelsächsischen Humangeographie in die Überlegungen ein: Statt der Fokussierung auf die Region gilt dort das Hauptaugenmerk der spezifischen Dialektik des Lokalen und Regionalen zum Globalen. Damit einher geht eine Relativierung des Regionsbegriffs: „Das Regionale wird zu einer Fallgruppe des Besonderen im dialektischen Verhältnis zum Globalen".?

Gemeinsam ist den neueren Diskussionsbeiträgen über alle humanwissenschaftlichen Disziplingrenzen hinweg, dass sie einem allgemeinen Trend weg von positivistischen und hin zu konstruktivistischen Interpretationsmustern folgen. ${ }^{8}$ Im Folgenden soll vor dem Hintergrund dieser spannenden Debatte der Regionsbegriff in der deutschen Raumordnung näher beleuchtet werden. Welche Konsequenzen ergeben sich für die raumplanerische Praxis und die angewandte raumwissenschaftliche Forschung aus dem gewandelten Regionenverständnis? Entspricht die Organisation der Regionalplanung den Anforderungen, die sich aus neueren Erkenntnissen in den Sozialwissenschaften ableiten lassen?

\section{Welchen Regionsbegriff verwendet die Raumordnung?}

Die „Region“ als identifizierbare Einheit ergibt sich primär aus der individuellen Perspektive des Betrachters, die Identifizierung einer regionalen Ebene hängt in höchstem Maße von der spezifischen Fragestellung einer Untersuchung ab. An dieser Stelle ist die Betrachtung des Begriffes folglich auf die raumordnerische und planungspolitische Diskussion zu beschränken. Insbesondere wird darauf verzichtet, das in den unterschiedlichsten Kontexten differierende Regionen-Verständnis anhand der nahezu unüberschaubaren Literatur umfassend aufzuarbeiten. Ebenso wenig tragen etymologische oder historische Ableitungen zur Erklärung der heutigen Verwendung dieses Schlagwortes bei. ${ }^{9}$ Schließlich entzieht sich der Begriff der „Region“ auch juristisch einer eindeutigen Bestimmung. Das Raumordnungsgesetz (ROG) setzt ihn voraus, ohne ihn näher zu erläutern. Losgelöst vom engeren Planungsrecht kommt Mecking generell zu der Erkenntnis, dass eine einheitliche juristische Terminologie von Region im deutschen Rechtskreis fehle. ${ }^{10}$ Auffindbar sei nur ein Über- und Durcheinander von rechtlich bestimmten räumlichen Einheiten mit planerischen oder administrativen Funktionen.

Die verbreitete Unsicherheit über das, was denn nun unter einer „Region“ zu verstehen sei, führt in der raumordnerischen Praxis nicht selten zu dem Argument, dass es vielleicht ja auch gar nicht so wichtig sei, ein einheitliches Begriffsverständnis $\mathrm{zu}$ entwickeln. Man konzentriert sich lieber auf die Lösung konkreter Problemstellungen, als sich mit abstrakten „Philosophien" zu beschäftigen. Die Begriffspluralität unreflektiert zu akzeptieren, hieße jedoch, vermeintliche Synonyme zu vermischen und die Gefahr nur scheinbar logischer Ableitungen durch den Wechsel der Begriffsinhalte herbeizuführen. ${ }^{11}$

Auch wenn der tatsächliche Sprachgebrauch in der räumlichen Planung terminologische Differenzierungen weitgehend ignoriert, erscheint es zweckmäßig, zumindest zwei Regionalbegriffe $\mathrm{zu}$ unterscheiden: einen deskriptiven und einen normativen. ${ }^{12}$ Im deskriptiven Verständnis wird nach bestehenden, „echten“ räumlichen Einheiten mittlerer Größenordnung gefragt. Deskriptive Regionsbestimmungen bauen auf einer Bestandsanalyse auf und bestimmen Gebiete, die entweder eine homogene Struktur („,homogene Regionen“) oder eine funktionale Verflechtung (,funktionale Regionen") aufweisen. Während homogene Regionen sich grundsätzlich auf eine beliebige Sachdimension beziehen können - diese wird in disjunkte Klassen unterteilt und auf bestimmte Gebiete projiziert -, stellen funktionale Abgrenzungen zumeist auf ökonomische Gesichtspunkte (Bezugs-, Absatzverflechtungen) oder auf die Daseinsgrundfunktionen (Wohnen, Arbeit, Bildung, Versorgung und Erholung) ab. Derart gefasste Regionen sollen also einen Lebens- und Aktionsraum widerspiegeln, innerhalb dessen die wesentlichen räumlichen Ausprägungen der Daseinsgrundfunktionen für seine Einwohner erfahrbar sind. 
Normative Regionen sind hingegen das Ergebnis politischer Entscheidungen. Gestaltungswille und Zielsetzung von Legislative und Exekutive bestimmen die räumliche und sachliche Dimension der „PlanungsRegion". Neben den bekannten administrativen Planungsregionen gibt es weitere normative Regionen. So wird beispielsweise im Raumordnungspolitischen Handlungsrahmen im Zusammenhang mit Regionalen Entwicklungskonzepten und Regionalkonferenzen die „Region“ als „Umsetzungsebene raumordnerischer Aktivitäten" verstanden. Gemeint sind aber weniger die Planungsregionen als vielmehr spezifische Räume, die sich über gemeinsame Strukturprobleme definieren. ${ }^{13}$

Die Tragweite einer Vermischung unterschiedlicher Regionsbegriffe zeigt sich exemplarisch, wenn aus dem postulierten Bedeutungszuwachs (deskriptiver) ökonomischer Regionen unvermittelt auf den Bedeutungszuwachs (normativer) Planungsregionen geschlossen wird. Wenn nach der vermeintlichen Entdeckung „leibhaftiger Regionen“, die eigenständiges Wirtschaftswachstum in Gang setzen konnten, die Bedeutung der "Region“ für staatliches Handeln als völlig unterschätzt angesehen wird. Oder wenn in einem „Europa der Regionen“, in dem sich die deutschen Länder als europäische „Regionen“ verstehen, die „Regionalisierungspolitik“ dieser Länder damit begründet wird, die „Regionen“ seien die Träger des Wettbewerbs im zukünftigen Europa. Hier werden unterschiedliche Inhalte mit dem synonym verwendeten Etikett "Region“ versehen. Die angesprochenen "Regionen“ beruhen auf einem passiv-beschreibenden Begriffsverständnis. Der direkte Bezug zur aktiv-gestalterischen Umsetzung verkennt den elementaren Unterschied zwischen räumlichem Beschreibungsobjekt einerseits und räumlichem Gestaltungsobjekt andererseits. ${ }^{14}$

Die Kongruenz von deskriptiven und normativen Regionen ist keineswegs selbstverständlich, sondern vielmehr von der politischen Willensbildung abhängig. Normative Regionen sollten dabei aus planerischer Sicht auf der Ermittlung deskriptiver Regionen aufbauen. Im politischen Raum und in der Öffentlichkeit sind (Planungs-) Regionen „also Gestaltungseinheiten, sie sind als politische Willensäußerung zu verstehen; es gibt sie nicht schon an sich“" ${ }^{15}$

Abbildung 1
Der Versuch, übereinstimmende Merkmale normativer Planungsregionen zu ermitteln, endet zumeist schon mit der lapidaren Feststellung, dass unter einer Region alle Raumeinheiten mittlerer Größe zwischen der kommunalen Ebene und der Landesebene verstanden werden können. Die geringen Gemeinsamkeiten entspringen der bewusst offen gehaltenen Vorgabe des $\$ 9$ ROG. Als Objekt politischer Entscheidungen können Planungsregionen keine endgültig definierten administrativen Einheiten sein. Sowohl Abgrenzung als auch Kompetenzen der Planungsregionen sind in der Vergangenheit mehrfach modifiziert worden. Sie werden auch zukünftig Gegenstand politischer Auseinandersetzungen sein und damit zur Disposition stehen.

Betrachtet man die tatsächliche Zuschneidung der regionalen Planungsräume in Deutschland (vgl. Abb. 1), so zeigt sich ein ausgesprochen uneinheitliches Bild.

Größe der Planungsregionen in Deutschland

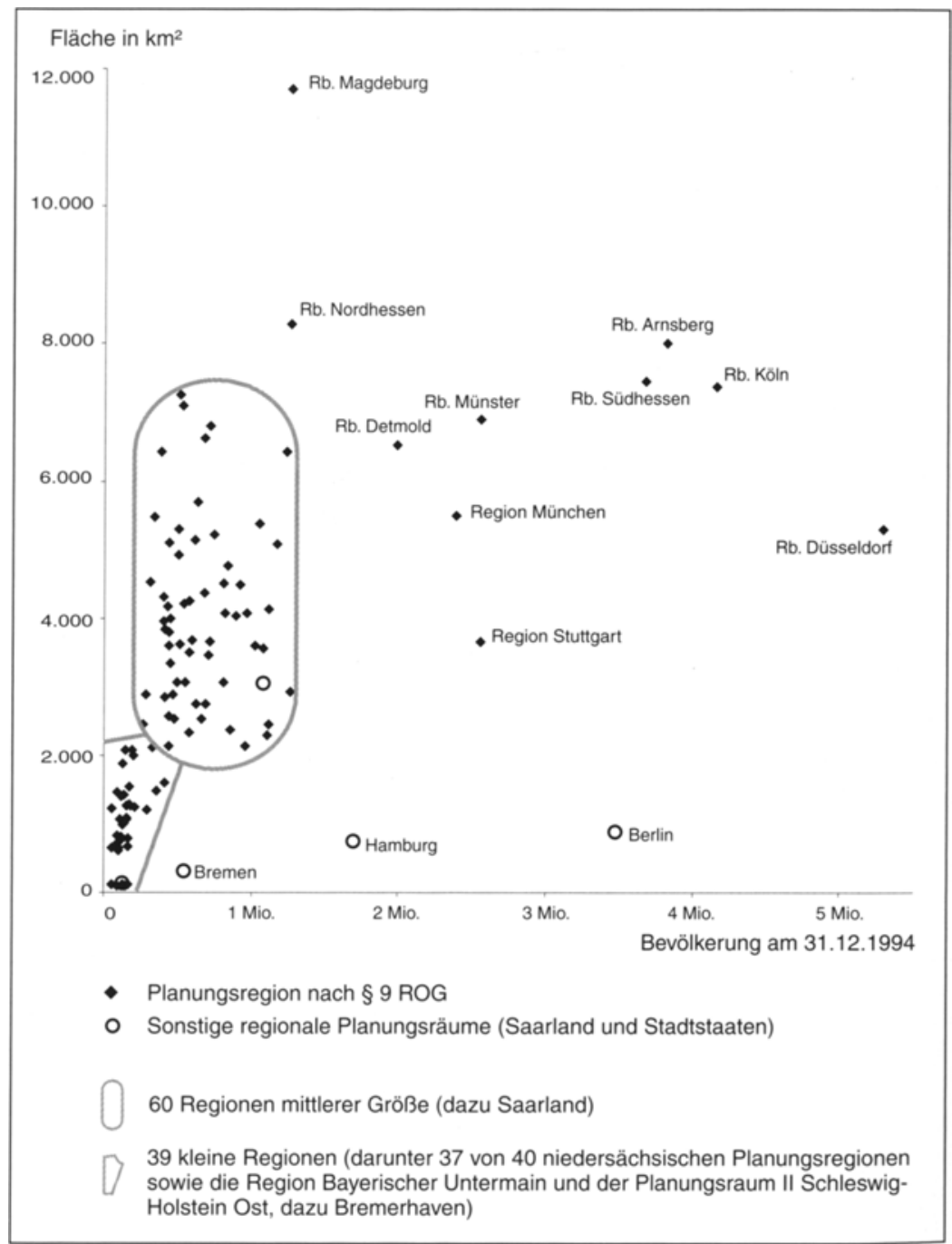

Quelle: Wiechmann (1998): Vom Plan zum Diskurs? - Baden-Baden, S. 333 Datengrundlage: BfLR: Laufende Raumbeobachtung 
Deutsche Planungsregionen weisen heute zwischen 51000 und 5,3 Mio. Einwohnern auf und umspannen ein Fläche von $62 \mathrm{~km}^{2}$ bis $11703 \mathrm{~km}^{2}{ }^{16}$ Manche Planungsregionen umfassen bis zu sechs Oberzentren, andere keines. Es ist offensichtlich, dass zwischen diesen "Regionen“ nur wenige Gemeinsamkeiten bestehen.

Versuche, die „Region“ aus planungsrelevanter Perspektive zu erfassen, lassen sich - über die Unterscheidung in normative und deskriptive Begriffe hinaus gliedern in differenzierende und synthetische Regionsbegriffe. Bei differenzierenden Regionsbegriffen wird die Unterscheidung einzelner Elemente, Strukturen oder Funktionen, die die Regionen charakterisieren, bezweckt. Bei synthetischen Regionsbegriffen steht hingegen das regionale System als Ganzes im Vordergrund. Sie sind sowohl Ausdruck der zunehmenden Interdependenz aller Akteure als auch der sinkenden Handlungsfähigkeit und Bedeutung administrativhoheitlicher Steuerungssysteme. Allerdings stellen sie damit höhere Anforderungen an das menschliche Denken. Die Bildung linearer Kausalketten und die Anwendung isolierten Fachwissens werden ganzheitlichen Betrachtungsweisen nicht gerecht. Die Frage, ob der Mensch - mithin auch der Planer - überhaupt fähig ist, angesichts der „Unzulänglichkeiten seines Denkens“ mit komplexen Systemen umzugehen, soll an dieser Stelle ausgeklammert werden. ${ }^{17}$

Die Klassifikation von Regionsbegriffen in Übersicht 1 gliedert Regionsbegriffe nach dem oben erwähnten Kriterium, wobei die differenzierenden Begriffe ihrerseits noch einmal in deskriptive und normative unterteilt werden. Außerdem wird der variierende räumliche Zuschnitt in die Betrachtung einbezogen. Neben Regionsbegriffen, die mit festen Abgrenzungen operieren, gibt es solche, die auf eine exakte territoriale Definition aus unterschiedlichen Motiven verzichten.

Übersicht 1

Klassifikation von planungsrelevanten Regionsbegriffen

\begin{tabular}{|c|c|c|c|}
\hline & \multicolumn{2}{|c|}{ Differenzierende Regionsbegriffe } & \multirow{2}{*}{$\begin{array}{c}\text { Synthetische } \\
\text { Regionsbegriffe }\end{array}$} \\
\hline & deskriptiv & normativ & \\
\hline $\begin{array}{l}\text { Territorial } \\
\text { definierte } \\
\text { Einheiten }\end{array}$ & $\begin{array}{l}\text { z.B. Raumordnungs- } \\
\text { region, Stadtregion }\end{array}$ & $\begin{array}{l}\text { z.B. Planungsregion, } \\
\text { Förderregion }\end{array}$ & \multirow{2}{*}{$\begin{array}{c}\text { z.B. } \\
\text { „Region der Zukunft“ }\end{array}$} \\
\hline $\begin{array}{l}\text { Nicht } \\
\text { territorial } \\
\text { definierte } \\
\text { Einheiten }\end{array}$ & $\begin{array}{l}\text { z.B. Wirtschaftsregion, } \\
\text { Kulturregion, } \\
\text { ökologische Region }\end{array}$ & $\begin{array}{l}\text { z.B. „Bio-Regio“, } \\
\text { „InnoRegio“ }\end{array}$ & \\
\hline
\end{tabular}

Quelle: (leicht verändert): Wiechmann: Vom Plan zum Diskurs? - Baden-Baden 1998, S. 134
Nach dieser Klassifikation, die im Sinne des eingangs Gesagten keinen Anspruch auf Vollständigkeit erheben kann, korrespondieren spezifische Perspektiven mit charakteristischen Gliederungsprinzipien. Deskriptiv-differenzierende Regionsbegriffe mit präziser Abgrenzung werden zumeist zum Zweck quantitativ-statistischer Analyse definiert. Beispiele sind die „Raumordnungsregionen" des BBR und die Boustedt'schen Stadtregionen. Aus der planungsrechtlichen Perspektive wird ein Land flächendeckend in ein Mosaik territorial definierter Planungsregionen aufgeteilt. Die strukturpolitische Herangehensweise neigt hingegen dazu, Hierarchien zu bilden: „benachteiligte“ Gebiete bedürfen einer besonderen Förderung, dynamische „Wachstumsregionen" tragen die gesamtwirtschaftliche Entwicklung. Das strukturpolitische Instrumentarium, etwa der europäische „Regionalfonds“ oder die nationale „Gemeinschaftsaufgabe zur Verbesserung der regionalen Wirtschaftsstruktur“, grenzt unterschiedlich förderwürdige bzw. förderbedürftige normative Förderregionen ab. Grundsätzlich führen pragmatische Erwägungen dazu, dass politischadministrative Entscheidungsträger normative Regionen bevorzugt mit festen Abgrenzungsmodalitäten verbinden. Ausnahmen stellen z.B. die vom Bundesforschungsministerium 1995 bzw. 1999 ausgeschriebenen Wettbewerbe „BioRegio“ und „InnoRegio“ dar. Ziel beider Wettbewerbe war bzw. ist die Verbesserung der bestehenden Fördermöglichkeiten durch eine regionale Vernetzung der wissenschaftlichen und wirtschaftlichen Aktivitäten. Dabei wurde die territoriale und institutionelle Organisation den teilnehmenden Regionen freigestellt.

Die Mehrzahl der wissenschaftlich motivierten Definitionen von Regionen fußt auf einem deskriptiven Begriffsverständnis. Sofern nicht statistische Berechnungen dies nahelegen, verzichten sie i.d.R. auf eine territoriale Abgrenzung, da diese fast immer funktionale Verflechtungen durchschneidet und arbiträre Entscheide voraussetzt. Aus sozio-kultureller Perspektive stellt sich der Staat als ein Nebeneinander von eng verflochtenen Regionen mit spezifischer - eben regionaler - „Identität“ dar. Dies können z.B. Kulturregionen oder Sprachräume sein. Es liegt auf der Hand, dass hier starre Abgrenzungen ebenso unangemessen sind wie bei der Definition von Regionen aus ökologischer Perspektive, bei der sich ein vergleichbares Nebeneinander von eng verflochtenen Ökosystemen ergibt. Das räumlich flexible Begriffsverständnis kommt auch bei einer ökonomischen Perspektive zu Tragen, die die Welt als Schauplatz eines interregionalen Wettbewerbs 
begreift. Deskriptive Wirtschaftsregionen können zwar räumlich erfasst werden, es kann ihnen eine Vielzahl von Eigenschaften zugeschrieben werden, eine Grenzziehung zwischen diesen unterläge jedoch immer einer gewissen Willkür. Wie bei sozio-kulturellen Abgrenzungen existieren Überschneidungsbereiche, Grenzen sind dynamisch, verändern sich im Zeitablauf. ${ }^{18}$

Synthetische Regionsbegriffe versuchen, eine fragmentarische Differenzierung zu vermeiden und die ganze Komplexität von Regionen in ihr Konzept zu integrieren. Danach besteht das Phänomen "Region“ als offenes, dynamisches System aus mehr als der Summe seiner Bestandteile. Nicht die trennenden, sondern die verbindenden Elemente, die gemeinsame Verantwortung stehen im Vordergrund. Vernetztheit, Interaktion und Interdependenz erhalten den Status von Schlüsselbegriffen. Die Unübersichtlichkeit komplexer Systeme, die Komplexität und Kontextualität des Sozialraumes machen diese jedoch schwerer fassbar. ${ }^{19}$ Synthetische Begriffe drohen entweder auf einer nomothetisch-abstrakten Metaebene zu verharren oder sie beschränken sich mehr oder weniger auf die ideographisch-deskriptive Thematisierung von Einzelfällen, wobei induktive Schlüsse kaum möglich sind.

Es ist daher aus regionalplanerischer Perspektive bis heute nicht gelungen, einen überzeugenden synthetischen Regionsbegriff zu entwickeln. Einen Schritt weiter sind Versuche, synthetische Begriffe auf globaler Ebene zu entwickeln. Mit Schlagworten wie „Weltgesellschaft", „Eine Welt" oder "Erdpolitik“ verbinden sich ganzheitliche, synthetische Weltbilder, die die nach wie vor vorherrschenden differenzierenden Weltbilder zunehmend ablösen. ${ }^{20}$ Für die Entwicklung eines synthetischen Regionsbegriffes bietet das Konzept der "nachhaltigen Raumentwicklung“ erste vielversprechende Ansätze. Es versucht sich einer ganzheitlichen Sicht der Region zu nähern, indem es den Netzwerk-Gedanken aufgreift und sowohl ökonomische als auch sozio-kulturelle und ökologische Aspekte berücksichtigt. Seit der 1998 erfolgten Novellierung des ROG gilt die nachhaltige Raumentwicklung als Leitvorstellung der Raumordnung, mithin auch der Regionalplanung. Ansätze zur Weiterentwicklung der Regionalplanung sind somit aufgefordert, sich an einem synthetischen Regionsbegriff im Sinne einer nachhaltigen Regionalentwicklung zu orientieren. In diese Richtung zielt beispielsweise der Wettbewerb „Regionen der Zukunft", den die Bundesforschungsanstalt für Landeskunde und Raumordnung (BfLR - heute Bundesamt für Bauwesen und Raumordnung, BBR) 1997 ausgeschrieben hat. Die territoriale und institutionelle Organisation wurde den Regionen auch hier freigestellt. „Fix“ war nur die Bedingung, dass die Regionen oder regionalen Initiativen überörtliche Ziele einer nachhaltigen Raum- und Siedlungsentwicklung verfolgen.

\section{Wie gestaltet sich das Verhältnis der regionalen und zur lokalen Raumplanung?}

Wie grenzt sich die regionale Ebene im Bereich der Raumplanung zur lokalen Ebene ab? In der Vergangenheit schienen die Fronten zwischen der Regionalplanung und der kommunalen Bauleitplanung relativ klar. Alles, was örtlichen Charakter hatte, galt als kommunal, alles, was überörtlichen Charakter hatte, wurde anderen Trägern zugewiesen. Müller-Ibold weist allerdings zu Recht darauf hin, dass die Stadt ,in ihrem Ursprung auf überörtlichen Funktionen begründet ist." 21 Insofern hatte Stadtplanung immer auch eine regionale Dimension, war die Grenze zwischen örtlicher und überörtlicher Planung immer fließend. Die Reichweite der örtlichen Aufgaben deckte sich dabei regelmäßig mit den Gemeindegrenzen. Mit dem "Ausufern“ der örtlichen Probleme über die Gemeindegrenzen hinweg werden die alten Zuständigkeiten infrage gestellt.

Prinzipiell bieten sich in einer derartigen Situation zwei Handlungsoptionen, die unterschiedlichen Politikmodi entsprechen, an: Zum einen könnte man im Sinne „hierarchisch-majoritärer“ Logik mit Hilfe einer kommunalen Gebietsreform den Versuch unterneh men, die Deckungsgleichheit zwischen den kommunalen Aufgaben und Grenzen wiederherzustellen, die Grenzen den Aufgaben anzupassen. Dadurch wäre die Kongruenz von Beteiligten und Betroffenen erneut gegeben. Diese Strategie wurde in der Vergangenheit (20er Jahre, 70er Jahre) wiederholt als „logischste“ Lösung verfolgt, und die Politikforschung kommt auch heute noch zu dem Schluss, dass die hierarchische Koordination gegenüber der Koordination auf freiwilliger Grundlage erhebliche Vorteile bietet. ${ }^{22}$ Dagegen sprechen aber schwerwiegende Einwände: Mit einer Verlagerung nach oben ginge unvermeidlich Bürgernähe verloren, die Größenordnung regionaler Gebietskörperschaften würde immer weniger überschaubar. Auf Seiten der betroffenen Kommunen muss von einem erheblichen Widerstand ausgegangen werden. Außerdem weisen die vielfältigen Probleme, denen sich die Kommunen heute ausgesetzt sehen, mindestens ebenso vielfältige, im Zeitablauf variierende räumliche Zuschnitte auf. Jede Problemstellung hat spezifische Betroffene, spezifische Adressaten und eine spezifische Reichweite. Ein für freiwillige Kooperationen notwendiges $\mathrm{Ma} \beta$ an Gemeinsamkeiten findet sich nur in Bezug auf Problemlösungen, nicht aber in Bezug auf Regionen. ${ }^{23}$ Diese Erkenntnis lässt das Konzept der 
territorial abgegrenzten Regionen insgesamt fragwürdig erscheinen. Neben der politisch kaum durchsetzbaren Gemeindegebietsreform bietet sich auch der Weg einer regionalen Gebietskörperschaft oberhalb der weiterhin bestehenden kommunalen Gebietskörperschaften an, also der Verlagerung der Aufgabe auf die erst zu schaffende nächsthöhere Entscheidungsebene. Dieses Regionalkreismodell - von Robert Schmidt schon in den 20er Jahren befürwortet - wird ergänzt durch eine kaum noch überschaubare Zahl von kommunalverfassungsrechtlichen Modellvorschlägen, die seit dem Zweiten Weltkrieg immer wieder diskutiert, aber mit wenigen Ausnahmen (z. B. Stadtverband Saarbrücken) aus unterschiedlichen Gründen auch wieder verworfen wurden. ${ }^{24}$

Angesichts dieser Schwierigkeiten drängt sich die Frage auf, ob „örtliche Aufgaben“ nicht auch über „örtliche Grenzen" hinweg denkbar sind. Richter fordert dazu auf, diese Begriffe inhaltlich zu überdenken, da sie durch die tägliche Lebensgestaltung der Bürger ohnehin bereits neubestimmt seien. ${ }^{25}$ Wenn örtliche Aufgaben solche Angelegenheiten seien, die sich üblicherweise aus dem Zusammenleben der Menschen auf engem Raum ergeben, die den Bürger in seinen alltäglichen Lebensbereichen berühren, dann bedürften diese auch im Falle einer überörtlichen Ausdehnung einer (inter-)kommunalen Bearbeitung. Der Logik eines „konsensualen Politikmodus“ folgend, böten daher individuelle interkommunale Lösungen grenzüberschreitender örtlicher Angelegenheiten eine Alternative zur regionalen Gebietskörperschaft. Nach Scharpf kennzeichnet dieses Politikverständnis, dass Entscheidungen von vornherein an einer Mehrzahl von Bezugseinheiten orientiert sind, es nicht auf die Mehrheit, sondern auf allseitiges Einverständnis ankommt, und Widerstrebende nicht zwangsweise zur Raison gebracht werden können. ${ }^{26}$ Aus kommunaler Sicht hat dies v.a. den Vorteil, dass in weitaus geringerem Maße Kompetenzen abgegeben werden müssen. Regionale Lösungen wären auf ganz konkrete Sachfragen beschränkt, die allgemeine Administration bliebe kommunal organisiert. In einer solchen von zahlreichen Kooperationen und Projekten geprägten Planungslandschaft wäre die Aufgabe der Regionalplanung keineswegs obsolet. „Wenn aus solchen Auslagerungsmaßnahmen kein zufälliges Konglomerat von jeweiligen Ad-hoc-Maßnahmen werden soll, wird regionale Abstimmung unerlässlich. “27 Der Tendenz von Zweckverbänden zur Entpolitisierung und institutionalisierten Eigeninteressen müssen regionale Strategien entgegengesetzt werden ${ }^{28}$ Bei flexiblen Strukturen, dezentraler Problembearbeitung und fließenden Grenzen ist die Funktion der Regionalplanung als ein Hauptknotenpunkt in einem regionalen Netzwerk Voraussetzung für eine integrale Regionalentwicklung.
Den beiden Optionen entsprechen Organisations- und Verfahrensinnovationen einerseits legalistischer und andererseits diskursiver Steuerungsversuche der Regionalplanung. Die Diskussion, welcher Weg erfolgversprechender ist, steht erst am Anfang. ${ }^{29}$ Wesentlich erscheint in diesem Zusammenhang, dass die oftmals bemühte Argumentationskette, nach der die größere, regionale Dimension kommunaler Aufgaben zwangsläufig einen Bedeutungsgewinn der Regionalplanung nach sich zieht, möglicherweise auf falschen Annahmen beruht - es handelt sich im Übrigen um eine unzulässige Vermischung deskriptiver Regionsbegriffe mit dem normativen Regionsbegriff der Planung -, denn zum einen führt eine Aufwertung der Region „nicht zwangsläufig zu einer Aufwertung der Regionalplanung, wenn sich stattdessen vermehrt themen(issue-)bezogene Netzwerke bilden ".30 Zum anderen macht die "Tatsache, dass eine örtliche Angelegenheit nicht mehr innerhalb bestehender Verwaltungsgrenzen gelöst werden kann, ... diese deshalb noch nicht automatisch zu einer überörtlichen oder regionalen Aufgabe ". ${ }^{31}$ Interkommunale Kooperationen haben insbesondere in Form von Zweckverbänden in der Vergangenheit schon oft ihre Leistungsfähigkeit bewiesen. Charakteristisch für die bisherige Zusammenarbeit war allerdings eine Interessenkongruenz, die bei den anstehenden Aufgaben selten vorausgesetzt werden kann.

Die Diskussion, welche Aufgaben noch interkommunal und welche Aufgaben wirklich regional zu lösen sind, ist unausweichlich. Die Antwort wird auch davon abhängen, ob es den Kommunen gelingt, auf freiwilliger Basis ihrer regionalen Verantwortung gerecht $\mathrm{zu}$ werden und auch politisch brisante Themen kooperativ zu lösen. Für Albers kann dies zum Prüfstein der Sachgerechtigkeit und Lebensfähigkeit kommunaler Selbstverwaltung deutscher Prägung werden. Sind die Kommunen nicht zu Kompromissen untereinander bereit, wird der Staat zwangsläufig sehr viel stärker tätig werden. ${ }^{32}$

\section{Was macht die Region so attraktiv?}

Die Unbestimmtheit des Regionsbegriffes hat seiner Popularität nicht geschadet. Im Gegenteil, die Begriffe "Region" und „Regionalisierung" entwickelten sich in einer Zeit, in der allenthalben ein Mangel an großen politischen Visionen beklagt wird, zu einer dehnbaren Formel für etwas Anderes, etwas Neues, etwas Besseres. Das Zauberwort „Region“ wurde zur Replik auf zahllose Herausforderungen unserer Zeit hochstilisiert: Es verspricht Schutz gegenüber nationalistischen und totalitären Ansprüchen, es ist die Antwort auf die Gefahren 
des Globalismus, mit ihm ist das provinzielle Kirchturmdenken zu überwinden; es wurde zur pauschalen Antithese für Bürokratie und Zentralismus. Viele machen sich diese suggestive Wirkung zu Nutzen: Ein Blick in eine beliebige Tageszeitung belegt die inflationäre Verwendung dieser Vokabeln. Verständlich, dass auch die Regionalplanung, die diesen Begriff schließlich schon vier Jahrzehnte in ihrem Namen führt, von dieser Entwicklung profitieren will. Der Versuch, ihn exklusiv zu besetzen, wäre aber von vornherein zum Scheitern verurteilt. Dafür ist ihre Rolle im öffentlichen Bewusstsein und ihre politische Macht zu begrenzt. Sie muss vielmehr ihr Augenmerk darauf lenken, dass nicht andere Akteure (Wirtschaftsressorts, Länder, Bezirksverwaltungen) den Regionsbegriff für sich reklamieren. Was aber macht die zweifelsfrei diffuse "Region“ im politischen Raum so attraktiv? Wie kommt es, dass ganz unterschiedliche Akteure für eine Regionalisierung eintreten? Ein wesentlicher Erklärungsansatz geht von der Annahme aus, dass die zentralen staatlichen Instanzen aus Eigeninteresse eine Verlagerung der Konfliktlösung "nach unten“ versuchten. ${ }^{33}$ Sie reagieren damit auf die zunehmende Überforderung mit politischen Konflikten. Die Region bietet dabei den Vorzug eines Forums abseits der klassischen Konfliktarenen, so dass hier ,zumindest symbolisch ein Neuanfang gesellschaftlicher Problembearbeitung versucht werden kann". ${ }^{34}$

Die Antwort ist aber auch in dem zu suchen, was Becker-Marx als die „Aggressivität des regionalen Prinzips" bezeichnet. Danach führte die Tatsache, dass die tradierte territoriale Gliederung des Raumes nicht mehr der räumlichen Wirklichkeit und der neuen Aufgabe staatlicher Daseinsvorsorge entsprach, zu einer Inadäquanz zwischen der sozial-räumlichen Frage und ihrer politischen Antwort. Daraufhin habe man nach den richtigen, gesellschaftsadäquaten Einheiten gefragt, und von nun an sei überall von der Region die Rede gewesen. Es sei deshalb gar keine Frage, dass dieser Begriff von Anfang an eine kritische Komponente gegenüber den alten Einheiten hatte. Die Region artikuliere das Unbehagen an der alten Ordnung und sei damit zu einem Synonym für eine neue, bessere Gliederung der Staaten geworden. Der Gegenentwurf zur überkommenen Ordnung sei von den etablierten Instanzen zunächst nur zögerlich als Gefahr wahrgenommen worden. Dennoch sei die Region bewusst als ein Torso konstruiert worden. Selbst eine bescheidene Erweiterung des schmal angelegten Kompetenzbereichs hätte ihre Kräfte aus den anderen, den alten Verwaltungskörpern ziehen müssen. Als sich die Länder gravierenden Folgerungen einer konsequenten Regionalisierung gegenüber sahen, hätten sie eilig zum Rückzug geblasen und ,verstaatlichte“ Modelle der
Regionalplanung präferiert. Die Region habe sich nicht in einer für die tradierten Verwaltungsformationen unschädlichen Weise in das System einarbeiten lassen; also sei sie aufgegeben worden. Der Stein sei solange zertrümmert worden, bis sich seine Reste leicht in die bestehenden Formationen haben einfügen lassen. ${ }^{35}$

Auch wenn man Becker-Marx in der negativen Einschätzung heutiger Regionalplanung vielleicht nicht ganz folgen mag, das reformistische, in seiner Anlage "aggressive" Element des Regionalen ist in der Tat ein Wesensmerkmal regionaler Bewegungen im weitesten Sinne. Aus oppositioneller Haltung heraus erweist sich die Unbestimmtheit des Regionsbegriffs durchaus als Vorteil. Man kritisiert das herrschende System, bleibt aber mit seinen eigenen Vorstellungen so vage, dass sich politische Mehrheiten mobilisieren lassen. Letztlich müssen Schlagworte erst bei ihrer Umsetzung mit Inhalten gefüllt werden. Das integrative Element ist die Negation des Etablierten, nicht die Umsetzung neuer Konzepte. Dieser strategische Vorteil von Opposition ist der Politikwissenschaft wohlvertraut.

Das „Regionale“ ist als Innovation auf die Übernahme von Kompetenzen etablierter Akteure angewiesen. Eine Weiterentwicklung regionaler Instanzen muss der Aggressivität des regionalen Prinzips Rechnung tragen. Das emanzipatorische, neue administrative Konstellationen erzwingende Element regionaler Entwicklung ist von eminenter politischer Brisanz und kann nicht ausschließlich mit planerischen Konzepten beantwortet werden. Im Grunde zeigt sich hier die gleiche Problematik wie bei der gescheiterten Länderneugliederung. War sie anfangs im Grundgesetz noch verbindlich vorgesehen, so steht sie nach zwei vergeblichen Vorstößen nicht mehr auf der Tagesordnung, obwohl an ihrer Zweckmäßigkeit aus fachlicher Sicht kaum ernsthafter Zweifel bestehen kann. Auch die Regionalplanung ist in den 70er Jahren bei dem ehrgeizigen Versuch gescheitert, als „Verwaltungsregion“ den Staatsaufbau zu reformieren. Wenn die politische Dimension nicht ausreichend beachtet wird, droht ähnlich ambitionierten Forderungen heute dasselbe Schicksal. Aus der Diskrepanz zwischen planerisch Wünschenswertem und politisch-administrativ Machbarem resultieren auf beiden Seiten Unverständnis und Frustration. Dies zeigt sich deutlich, wenn aus planerischer Perspektive argumentiert wird, die Region sei „die einzig zutreffende Antwort auf die sich auszubildende räumliche Organisationsform der Gesellschaft. Sie zu schaffen, musste eine Selbstverständlichkeit der planenden Verwaltung sein. Als 'Planungsregion' verkümmerte freilich alsbald die dem gesellschaftlichen Bewusstsein adäquate 'Raumorganisation'. Den Regionsbewohner nicht zum 
Regionsbürger zu machen, zeugte von der Unfähigkeit der alten Verwaltungsordnung, mit den neuen räumlichen Umgriffen der Bürger umzugehen ". ${ }^{36}$ Zwischen der alten Verwaltungsordnung und dem regionalplanerisch als notwendig Erkanntem bestehen scheinbar unüberbrückbare Differenzen. Umfassende Kompetenzerweiterungen für die Regionalplanung erzwingen einen neuen Verwaltungsaufbau. Entsprechende Forderungen allein planerisch zu begründen, hieße das komplexe Beziehungsgeflecht staatlichen Handelns auszublenden.

\section{Gibt es heute überhaupt eine regionale Ebene in Deutschland?}

Es stellt sich die Frage, ob man heute überhaupt von einer regionalen Ebene in Deutschland sprechen kann. Priebs verneint dies mit Blick auf die bunte Vielfalt administrativer Institutionen, die sich zwischen Gemeinde- und Landesebene tummele: „Praktisch bedeutet diese Zersplitterung jedoch, dass es die regionale Ebene in Deutschland - im Sinn einer leistungsfähigen und politisch legitimierten Verwaltungs- und Planungseinheit 'aus einem Guss' - nicht gibt" ${ }^{37}$ Inhaltlich kommt Danielzyk zu dem gleichen Ergebnis. Um den Eindruck zu vermeiden, es handele sich dabei um „politisch handlungsfähige, homogene Einheiten“, spricht er allerdings bewusst von einer (durchaus existenten) "regionalen Ebene“, statt von (nicht existenten) „Regionen“. ${ }^{38}$

Regionsbegriffe sind zwangsläufig das Ergebnis bewusster inhaltlicher Akzentuierungen, da sie auf einer starken Reduktion der komplexen Wirklichkeit beruhen. Je nach Standpunkt des Betrachters gelangen sie $\mathrm{zu}$ durchaus widersprüchlichen Resultaten. Gleichwohl sind sie wissenschaftliche Konstrukte und als Nominaldefinitionen nicht falsifizierbar. Hettner hat schon in den 20er Jahren darauf hingewiesen, dass es keine unzweideutige, objektiv zu bevorzugende Einteilung der Erdoberfläche geben könne. ${ }^{39}$ Alle geographischen Gliederungsgrenzen sind weder richtig noch falsch, vielmehr ist nach ihrer Zweckmäßigkeit im jeweiligen Kontext $\mathrm{zu}$ fragen. Gerade unter dem Gesichtspunkt planerischer Gestaltung fehlen aber überzeugende Regionsbegriffe, die die komplexen Regionalstrukturen adäquat abzubilden vermögen.

Die Aufteilung der Länder in Planungsregionen stellt folgerichtig eines der klassischen Streitthemen regionaler Planung dar. Es wurde bereits erläutert, dass es sich hier um die Ausweisung normativer, nicht deskriptiver Regionen handelt. Eine solche Abgrenzung ist also auf ihre Zweckmäßigkeit hin zu überprüfen, sie kann aber nicht "richtig“ oder "falsch" sein. Die Ermittlung deskriptiver Regionen dient dabei regelmäßig als Basis für die einer politischen Logik folgenden Ausweisung von Planungsregionen. Regionseinteilungen sind also „an ein Substrat in Form von Arealen und realen Funktionsbereichen gebunden". ${ }^{40}$ Je ausgeprägter die räumliche Kongruenz der relevanten Funktionen und Strukturen bei der vorgeschalteten, deskriptiven Abgrenzung homogener oder funktionaler Regionen ist, umso naheliegender ist es, diese deskriptiv ermittelten Regionen normativ nachzuvollziehen. Daraus abgeleitet ist die Idee von der Region als „Ding an sich“, als organisatorischer Einheit, die mit wissenschaftlichen Methoden objektiv abgegrenzt werden könne.

Die vergebliche Suche nach dieser "wahren" Region hat jedoch klargemacht, dass es die Region an sich nicht gibt. „So wenig wie es eine natürliche, richtige, wahre Sprache gibt, so wenig gibt es eine natürliche, richtige, wahre, in der Wirklichkeit selbst eindeutig vorgezeichnete Regionalisierung oder Region. Anders gesagt, hinter Regionen und Regionalisierungen stecken Interessen und Programme. ${ }^{41}$ Die Kongruenz von Arealen und Bereichen ist nicht vorgegeben, sondern das Resultat einer - insoweit durchaus willkürlichen - Auswahl bestimmter Merkmale. Alle Regionsbegriffe berücksichtigen nur einen selektiven Ausschnitt von Kriterien, blenden dabei aber zwangsläufig zahlreiche andere aus: ,A region is not an object, ... it is an intellectual concept, an entity . . created by the selection of certain features ... and by the disregard of certain others" ${ }^{42}$ Konsequenterweise äußerte Hartshorne schon 1939: „... we not only have not yet discovered and established regions as real entities, but we have no reason ever to expect to do so". ${ }^{43}$

Das Verständnis von Regionen, auch von raumordnerischen Regionen, sollte sich lösen von der missverständlichen Vorstellung eines Landstrichs mit festen Grenzen. Hard fordert dazu auf, Regionen nicht als physisch-materielle Wirklichkeiten (oder RaumGesellschafts-Komplexe) an der Erdoberfläche aufzufassen, sondern sie erst einmal als Konstrukte und Bestandteile der sozialen Kommunikation zu betrachten - oder auch als Zeichen in Texten. „Dort sind sie jedenfalls leichter zu finden als zum Beispiel in der materiellen Wirklichkeit an der Erdoberfläche, wo man schon so oft so vergeblich nach ihnen gefahndet hat, nicht zuletzt in meiner eigenen Disziplin, der Geographie“."44 Die "Region" als zu entdeckende, objektive Gliederungseinheit des Raumes ist ein Mythos. „Die 'Wahre Region' als ontologisch determinierte Einheit ist eine Fiktion, für die Praxis belanglos, als wissenschaftliche Fragestellung (zumindest in empirischen Kontexten) untauglich, und damit auch als Begriff der Objektsprache überflüssig. “45 
Einheitliche Abgrenzungskriterien für alle Regionen kann es nicht geben kann. In vielen Fällen gibt es zwischen der nach der politischen Systemlogik erfolgten Regionsabgrenzung und der planerisch wünschenswerten Orientierung an sozioökonomischen Verflechtungen erhebliche Widersprüche. Für die Zukunft wird entscheidend sein, ob es gelingt, die administrativ definierten Regionen für problembezogene Netzwerke zu öffnen. Dabei muss jedoch die Frage gestellt werden, ob überhaupt starre Regionsabgrenzungen erforderlich sind, ob nicht flexible, problemorientierte Abgrenzungen mit einzelnen Überschneidungen den zukünftigen Herausforderungen eher gerecht werden. Diesgerichtete Überlegungen sind nicht neu. In Rheinland-Pfalz hat man seit 1967 Erfahrungen mit Überschneidungen von Planungsregionen gesammelt. Der Landtag folgte damals schon dem modern anmutenden Konzept der Region als dem dynamischen Element der Staats- und Gesellschaftsordnung im Gegensatz zur statischen Verwaltungsorganisation. Einem flexiblen Regionenverständnis sind in jüngster Zeit auch die Bemühungen um eine Intensivierung der regionalen Zusammenarbeit in den Räumen Hamburg und Bremen verpflichtet. ${ }^{46}$

Die verbreitete und sowohl für die empirische Forschung als auch für die Administration praktische Vorstellung von Raum als einem Stück Land mit festen Grenzen wird der heutigen Dynamik räumlicher Prozesse selten gerecht. Weder ökonomische noch soziale noch ökologische Beziehungsgefüge konstituieren eine fest abgrenzbare Region. Nach Krätke bestehen Regionen aus einem Zusammenhang mehrerer Orte und Standorte, die ein funktionales Beziehungsgefüge bilden und ein Interaktionsfeld wirtschaftlich-sozialer Akteure darstellen. Diese seien ,jedoch in der Regel nicht eindeutig abgrenzbar, so dass man zum Zwecke empirischer Regionalanalysen häufig wieder eine 'territoriale’ Kompromisslösung akzeptiert ... ; dabei sollte jedoch eine naive Verwendung territorialer Regionsbegriffe vermieden werden". ${ }^{47}$

Trennt man sich von der fälschlichen Annahme „wahrer" Regionen und akzeptiert die Region als intellektuelles Konzept, so ist es folgerichtig anzunehmen, dass ein beliebiger Ort auf der Erdoberfläche unendlich vielen "Regionen" angehört, ein beliebiger Raum daher auch „multiregional“ definiert werden kann. Im Rahmen einer problembezogenen Neudefinition der Regionalplanung sollte der (deskriptive) Regionsbegriff daher nicht als abgegrenzter physischer Ausschnitt der Erdoberfläche verstanden werden, sondern als sozioökonomischer Verflechtungsraum, der sich als lose verbundenes Netzwerk privater und öffentlicher Institutionen in erster Linie durch die gegenseitige Wahr- nehmung der in ihm agierenden Akteure definiert. Ein kommunikations- und akteursbezogener Begriff verlangt aber eine variable, ,issue “-bezogene Abgrenzung, die sich an den zu lösenden Sachverhalten und den beteiligten bzw. den betroffenen Akteuren orientiert. Das wiederum setzt voraus, dass ein territorial definierter Raumausschnitt mehreren "Regionen“ gleichzeitig angehören kann - es entstehen gleichsam „multiregionale Räume“.

\section{Was heisst das für Raumforschung und Raumordnung?}

Welche Konsequenzen bringt nun das skizzierte konstruktivistische, akteursbezogene Verständnis des Regionsbegriffes mit sich? Ist der Begriff überhaupt geeignet, die Weiterentwicklung der Raumordnung an der Schwelle zum 21. Jahrhundert voranzutreiben? Ist das Verständnis der Region als "Container" nahtlos in ein postmodernes resp. postfordistisches Verständnis als

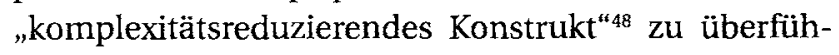
ren?

Für die Raumwissenschaft bedeutet die Relativierung des Regionsbegriffes zunächst einmal, dass sie sich verstärkt sozialwissenschaftlichen Gesellschaftstheorien, auf die sich die Mehrzahl der einschlägigen Autoren bezieht, zuwenden muss. Die pragmatische, bestenfalls mit Theorien mittlerer Reichweite operierende Raumwissenschaft muss wieder verstärkt den Dialog mit ihren Grundlagendisziplinen suchen. Soziologische, politikwissenschaftliche, ökonomische und geographische Theorieansätze können sich auf dem Gebiet der Raumwissenschaften gegenseitig befruchten und so das grundsätzlich zu konstatierende Theoriedefizit abbauen. Ein technisches Planungsverständnis, das den räumlichen Bezug als fixe Rahmenbedingung, als "Handlungsfolie“ begreift, wird den anstehenden Herausforderungen nicht gerecht. Akteursorientierte Begriffe und Theorieansätze der Regionalentwicklung sind stärker als bisher in dem Mittelpunkt der Betrachtung zu rücken. Regionale Akteure, Netzwerk, Lernende Region, Kooperation und Diskurs sind nur einige der Schlüsselbegriffe einer disziplinenübergreifenden Diskussion, die erst an ihrem Anfang steht. Die Bemühungen um eine "Theorie der Region" müssen daher in einem engen Zusammenhang mit den Bemühungen um eine „Theorie kooperativer Planungsverfahren" gesehen werden.

Kestermann hat in einem lesenswerten Aufsatz versucht, anhand phänomenologischer Betrachtungen Ansätze für die Überwindung der schmerzlichen Theorielücke im Bereich der kooperativen Verfahren in der Raumordnung anzudeuten. ${ }^{49}$ Danach verspreche eine 
Übersicht 2

Theorieansätze eines raumwissenschaftlichen Regionsbegriffs

\begin{tabular}{|l|c|c|}
\hline \multicolumn{1}{|c|}{$\begin{array}{c}\text { Theoretische Perspektiven } \\
\text { (Auswahl) }\end{array}$} & $\begin{array}{c}\text { Theorieansätze } \\
\text { (Beispiele) }\end{array}$ & $\begin{array}{c}\text { Vertreter } \\
\text { (Beispiele) }\end{array}$ \\
\hline $\begin{array}{l}\text { 1. Planungstheoretische } \\
\text { Ansätze }\end{array}$ & $\begin{array}{c}\text { Communicative Planning, } \\
\text { Neue Planungskultur, } \\
\text { Theorie der Politikverflechtung }\end{array}$ & $\begin{array}{c}\text { Healey, Friedmann } \\
\text { Selle, Fürst, } \\
\text { Benz, Scharpf }\end{array}$ \\
\hline $\begin{array}{l}\text { 2. Politisch-ökonomische } \\
\text { Gesellschaftstheorien }\end{array}$ & $\begin{array}{c}\text { Regulationstheorie, } \\
\text { Theorie der Strukturierung }\end{array}$ & $\begin{array}{c}\text { Lipietz, Amin, Krätke } \\
\text { Giddens, Werlen }\end{array}$ \\
\hline $\begin{array}{l}\text { 3. Netzwerktheorien } \\
\text { Ohonomische Netzwerktheorien, } \\
\text { Politische Netzwerktheorien }\end{array}$ & $\begin{array}{c}\text { Camagni, Cooke, Morgan, } \\
\text { Grabher, Mayntz }\end{array}$ \\
\hline $\begin{array}{l}\text { Handelns } \\
\text { 5. Normative theoretische } \\
\text { Ansätze }\end{array}$ & $\begin{array}{c}\text { Rational Choice Theory, } \\
\text { Spieltheorie }\end{array}$ & $\begin{array}{c}\text { Coleman, Buchanan } \\
\text { Axelrod }\end{array}$ \\
\hline
\end{tabular}

Vielzahl von Theorieansätzen aus den verschiedensten Disziplinen, zumindest für einzelne Aspekte und Fragestellungen fruchtbar zu sein. Aus planungswissenschaftlicher Perspektive hat auch eine "Theorie der Region" auf diese Vielfalt theoretischer Ansätze zurückzugreifen. Von besonderem Interesse werden in diesem Zusammenhang neben im engeren Sinne planungstheoretischen Ansätzen politisch-ökonomische Gesellschaftstheorien, Netzwerktheorien, Theorien rationalen Handelns und normative theoretische Ansätze nachhaltiger Stadt- und Regionalentwicklung sein (vgl. Übersicht 2).

Das gewandelte Regionenverständnis hat selbstverständlich auch Konsequenzen für die raumplanerische Praxis. Zwischen dem flexiblen, problem- und aufgabenorientierten Regionsbegriff und der aus pragmatischen wie juristischen Gründen wohl auch weiterhin unumgänglichen territorialen Abgrenzung normativer Planungsregionen bestehen Widersprüche. Zukünftig ist v.a. zu klären, wie beide Regionsbegriffe parallel Anwendung finden können, ob eine effektive Kombination von territorialen Planungsregionen und dynamischen "Aufgaben-Regionen" denkbar ist. Die Realisierungschancen der hier angedeuteten akteursbezogenen Antwort auf die problematische Abgrenzungsfrage sind noch weitgehend ungeklärt.

\section{Schlussbemerkung}

Eine interdisziplinäre Debatte über das Wesen der allgegenwärtigen „Region“ ist längst überfällig. Die in der Raumplanung verbreitete alltagssprachlich-naive Verwendung des Begriffes muss vor dem Hintergrund der neuen Maxime einer nachhaltigen Raumentwicklung abgelöst werden durch einen synthetischen Regionsbegriff, dessen Eckpunkte sich bereits schemenhaft andeuten. Im Kern geht es darum, sich von dem traditionellen, auf territoriale Abgrenzungen basierenden
Verständnis von „Region“ zu lösen. Es spricht sehr viel dafür, den Regionsbegriff nicht als physischen Ausschnitt der Erdoberfläche (miss)zu verstehen, sondern als sozio-ökonomischen Verflechtungsraum zu betrachten, der sich als lose verbundenes Netzwerk durch die gegenseitige Wahrnehmung der in ihm agierenden Akteure definiert. Der Regionalplanung käme in einem solchen zeitlich instabilen Netzwerk die Funktion eines Hauptknotenpunktes zu. Damit bietet sich die Chance, die administrativ definierten Regionen für problembezogene Netzwerke zu öffnen, ohne dass die „Aggressivität des regionalen Prinzips“ zu einer reflexartigen Blockade aller Reformanstrengungen durch etablierte Verwaltungseinheiten führt.

Der Wandel des Begriffsverständnisses, die zeitlich und räumlich instabile Anlage regionaler Netzwerke hat zumindest im Bereich der Raumplanung - den konventionellen landeskundlichen Regionsbegriff obsolet werden lassen. Er wird abgelöst durch ein Begriffsverständnis, dass neue Chancen bietet und neue Fragen aufwirft. Wie werden Regionen "gemacht"? Welchen Mustern folgt die Reifikation von Regionen? Wie werden solche Regionen beplant und gemanagt? Kann man überhaupt mittels instabiler Netzwerke den Raum "planen"? Die ontologische Region als „Ding an sich“ ist tot. Die Raumwissenschaft ist aufgefordert, ein neues Regionenverständnis zu entwickeln, ein Verständnis, das die Konjunktur des Regionalen für die Raumplanung nutzbar macht.

\section{Anmerkungen}

*) Überarbeitete Fassung eines Beitrags zum FRU-FörderpreisWettbewerb 1999. Dieser beruht auf Untersuchungen des Autors im Rahmen seiner Dissertation (vgl. Wiechmann, Th. (1998): Vom Plan zum Diskurs? Anforderungsprofil, Aufgabenspektrum und Organisation regionaler Planung in Deutschland. - Baden-Baden)

(1)

Vgl. Brunn, G. (Hrsg.) (1996): Region und Regionsbildung in Europa. Konzeptionen der Forschung und empirische Befunde. - Baden-Baden

(2)

Müller, B. (1994): Von räumlicher Koordination zu regionaler Kooperation. Perspektiven der Regionalplanung in der Bundesrepublik Deutschland. In: Akademie für Raumforschung und Landesplanung (Hrsg.): Regionalplanertagung Sachsen. = Arbeitsmaterial Nr. 205, Hannover, S. 127 
(3)

Blotevogel, H.H. (1996): Auf dem Wege zu einer "Theorie der Regionalität“: Die Region als Forschungsobjekt der Geographie. In: Brunn, G. (Hrsg.), a.a.O., S. 44

(4)

Vgl. Benz, A; Holtmann, E. (Hrsg.) (1998): Gestaltung regionaler Politik. Empirische Befunde, Erklärungsansätze und Praxistransfer, - Opiaden, sowie Ellwein, Th, Mitteistraß, J. (Hrsg.) (1996): Regionen, Regionalismus, Regionalentwicklung. - Oldenburg

(5)

Vgl. Weichhart, P. (1996): Die Region - Chimäre, Artefakt oder Strukturprinzip sozialer Systeme? In: Brunn, G. (Hrsg.), a.a.O., S. $25-43$

(6)

Vgl. Blotevogel, H.H. (1996) a.a.O

(7)

Krüger, R. (1998): Globalisierung und Regionalisierung als Thema der Regionalforschung - insbesondere unter dem Blick einer „New Regional Geography“. In: Berichte zur deutschen Landeskunde, Heft 1, S. 37-46

(8)

Vgl. Miggelbrink, J.; Wardenga, U, (1998): Rezensionsaufsatz zu: Brunn, G. (1996) a.a.O. In: Berichte zur deutschen Landeskunde, Heft 1, S. 69

(9)

Das Fremdwort „Region“ wurde im 15. Jahrhundert aus lat. „regio“ = „Richtung, Gegend, Bereich, Gebiet“ entlehnt. Dieses geht auf lat. "regere" = ,gerade richten, lenken, herrschen“ zurück. Der historischen Herkunft nach ist der Begriff „Region“ ein Element stadtrömischer Kommunalverfassung: Unter Augustus wurde Rom in 14 Regionen, also Stadtviertel, aufgeteilt.

(10)

Vgl. Mecking, Ch. (1995): Die Regionalebene in Deutschland. Begriff, Institutioneller Bestand, Perspektiven, Stuttgart u.a., S. 62

(11)

Vgl. Boesch, M. (1989): Engagierte Geographie. Zur Rekonstruktion der Raumwissenschaft als politikorientierte Geographie. In: Erdkundliches Wissen, Heft 98, Stuttgart, S. 43

(12)

Vgl. Lange, K. (1968): Die Organisation der Region. - Siegburg, S. $8 \mathrm{ff}$.

(13)

Vgl. Bundesministerium für Raumordnung, Bauwesen und Städtebau (Hrsg.) (1995): Raumordnungspolitischer Handlungsrahmen. Beschluß der Ministerkonferenz für Raumordnung in Düsseldorf am 8. März 1995, Bonn, S. I

(14)

Vgl. Lange, K. (1968), a.a.O., S. 9
(16)

Vgl. Wiechmann, Th. (1998), a.a.O., S. $330 \mathrm{ff}$.

(17)

Vgl. Dörner, D. (1996): Die Logik des Misslingens. Strategisches Denken in komplexen Situationen, veröff. August 1992, 56.-64. Tsd. April 1996, Reinbek bei Hamburg, insbesondere S. 288 ff.

(18)

Vgl. Krätke, S. (1995): Stadt, Raum, Ökonomie. Einführung in aktuelle Problemfelder der Stadtökonomie und Wirtschaftsgeographie. - Basel, Boston, Berlin, S. 9 f.

(19)

Vgl. Heymann, Th. (1989): Komplexität und Kontextualität des Sozialraumes. = Erdkundliches Wissen, Heft 95, Stuttgart

(20)

Vgl. Stiftung Entwicklung und Frieden (Hrsg.) (1991): Globale Trends, Daten zur Weltentwicklung. - Bonn, S. 32 ff.

(21)

Müller, Ibold, K. (1996): Einführung in die Stadtplanung, Bd. 2: Leitgedanken, Systeme und Strukturen. - Stuttgart, Berlin, Köln, S. 130

(22)

Vgl. zu den unterschiedlichen Politikmodi: Benz, A.; Scharpf, F.W.; Zintl, R. (1992): Horizontale Politikverflechtung. Zur Theorie von Verhandlungssystemen. In: Schriften des Max-Planck-Instituts für Gesellschaftsforschung, Bd. 10, Frankfurt, New York; insbesondere die Beiträge von Scharpf, F.W.: Kapitel 1, Einführung: Zur Theorie von Verhandlungssystemen, S. $11 \mathrm{ff}$. und Zintl, R.: Kapitel 4, Kooperation und Aufteilung des Koordinationsge winns bei horizontaler Politikverflechtung, S. 97

(23)

Vgl. Fürst, D. (1994): Stadt und Region. In: Mäding, H. (Hrsg.): Stadtperspektiven, DIFU-Symposium 1993. In: DIFU-Beiträge zur Stadtforschung, Bd. 10, S. 50

(24)

Vgl. die ausführliche Darstellung der Stadt-Umland-Modelle bei Lange, K. (1968), a.a.O., S. 59 ff. und Trümper, A. (1982): Raumbe zogene Planung im Großstadt-Umland-Bereich. In: Schriftenrei he der Gesellschaft für Regionale Strukturentwicklung, Bd. 8, S. $81 \mathrm{ff}$.

(25)

Vgl. Richter, K. (1992): Kommunale Planungshoheit und regiona le Verantwortung. In: ARL (Hrsg.): Notwendigkeiten, Möglichkeiten und Grenzen interkommunaler Zusammenarbeit. Regional planertagung 1991 vom 6 . bis 8 . November 1991 in Überlingen. In: Arbeitsmaterial Nr. 191, Hannover, S. 43

(26)

Vgl. Scharpf, F.W. (1992), a.a.O., S. 11 f. 
(27)

Albers, G. (1994): Regionales Planungsmanagement und kommunale Planungshoheit - vorprogrammierter Konflikt oder Chance zur Kooperation? Manuskript zur Fachtagung „Kommunalpolitische Informationstagung" der DASL am 21. März 1994 in München, S. 1

(28)

Vgl. Fürst, D. (1994), a.a.O., S. 50

(29)

Vgl. Wiechmann, Th. (1998), a.a.O., S. 150

(30)

Fürst, D. (1993): Von der Regionalplanung zum Regionalmanagement? In: Die Öffentliche Verwaltung, Heft 13, S. 557

(31)

Richter, K. (1992): a.a.O., S. 55 f.

(32)

Vgl. ebenda, S. 4 sowie Müller-Ibold, K. (1996), a.a.O., S. 23

(33)

Vgl. Danielzyk, R. (1994): Regionalisierung der Ökonomie - Regionalisierung der Politik in Niedersachsen: Zur Aktualität geographischer Regionalforschung. In: Berichte zur deutschen Landeskunde, Bd. 68, Heft 1, S. 88

(34)

Fürst, D. (1993): Der Region auf die Sprünge helfen. In: Passage, Heft 1, S. 25

(35)

Vgl. Becker-Marx, K. (1981): Das Schicksal der Region in der Bundesrepublik Deutschland. In: Mannheimer Geographische Arbeiten, Heft 12, S. 85 ff.

(36)

Ante, U.; Wille, V. (1991): Raumordnung, Landes- und Regionalplanung. Versuch eines Befundes aus zeitlicher und fachlicher Distanz. In: ARL (Hrsg.): Zur geschichtlichen Entwicklung der Raumordnung, Landes- und Regionalplanung in der Bundesrepublik Deutschland. In: Forschungs- und Sitzungsberichte der ARL, Band 182, Hannover, S. 436

(37)

Priebs, A. (1996): Stärkung der Region und Straffung der regionalen Verwaltung - Gebot der Stunde und Herausforderung für die Angewandte Geographie! In: Standort, Heft 2, S. 47

(38)

Vgl. Danielzyk, R. (1994), a.a.O., S. 104

(39)

Vgl. Hettner, A. (1927): Die Geographie, ihre Geschichte, ihr Wesen, ihre Methoden. - Breslau

(40)

Boesch, M. (1989), a.a.O., S. 68
(41)

Hard, G. (1994): Regionalisierungen. In: Wentz, M. (Hrsg.): Region. Die Zukunft des Städtischen, Bd. 5, Frankfurt, New York, S. 54

(42)

Whittlesey, D. (1954): The Regional Concept and the Regional Method. In: James, P.E.; Jones, L.F. (Ed): American Geography Inventory and Prospect. - Syracuse NY, S. 30

(43)

Zitiert nach Wtt, W. (1960): Stadtlandschaft, Stadtregion, Regionalplanung. Kritische Bemerkungen zur Bedeutung regionaler Begriffe. In: ARL (Hrsg.): Stadtregionen in der Bundesrepublik Deutschland, Raum und Bevölkerung 1. In: Forschungs- und Sitzungsberichte der ARL, Bd. XIV, Hannover, S. 97

(44)

Hard, G. (1994), a.a.O., S. 54

(45)

Boesch, M. (1989), a.a.O., S. 65

(46)

Solche Ansätze stehen im Einklang mit den aktuellen Forschungsergebnissen der Wirtschaftsgeographie [vgl. Krätke, S. (1995):, a.a.O., S. 77 ff.]. Diese richtet ihr Augenmerk in jüngster Zeit verstärkt auf die Verflechtungsstrukturen innerhalb und zwischen (deskriptiven) Wirtschaftsregionen. Dahinter steht die Hypothese, dass die Art und Intensität der Verflechtungen zwischen den regionalen Akteuren verschiedener Regionen und innerhalb derselben die unterschiedliche Entwicklungsdynamik der Regionen prägt. Aufgegriffen wurde dieser Gedanke vom Konzept des „regionalen Produktionsmilieus“, das von französischen Wissenschaftlern der "Group de Recherche Europeen sur les Milieux Innovateurs" (GREMI) Mitte der 80er Jahre entwickelt wurde. Die Region wird hier nicht mehr als passiver Behälter angesehen, in dem mehr oder weniger innovative Unternehmen als isolierte Elemente der lokalen Wirtschaft angesiedelt sind, sondern als der Rahmen eines aktiven Milieus, aus dem heraus innovative Entwicklungen erzeugt und vorangetrieben werden und das auf vielfältigen Kooperationsformen zwischen den privaten und öffentlichen Akteuren beruht, welche die Region selbst als „kollektiven Akteur“ erscheinen lassen.

(47)

Krätke, S. (1995), a.a.O., S. 10

(48)

Vgl. Miggelbrink, J.; Wardenga, U. (1998), a.a.O., S. 69

(49)

Kestermann, R. (1997): Kooperative Verfahren in der Raumplanung. Phänomenologische Betrachtungen. In: Adam, B. (Hrsg.): Neue Verfahren und kooperative Ansätze in der Raumplanung. In: RaumPlanung spezial 1997, S. 50-78

Dr. Thorsten Wiechmann

Institut für ökologische Raumentwicklung e.V.

Weberplatz 1

01217 Dresden

E-Mail: Thorsten.Wiechman@mailbox.tu-dresden.de 